\title{
On Zudilin's q-question about Schmidt's problem
}

\author{
Victor J. W. Guo ${ }^{1}$ and Jiang Zeng ${ }^{2}$ \\ ${ }^{1}$ Department of Mathematics, East China Normal University \\ Shanghai 200062, People's Republic of China \\ jwguo@math.ecnu.edu.cn, http://math.ecnu.edu.cn/ ${ }^{\sim}$ jwguo \\ ${ }^{2}$ Université de Lyon; Université Lyon 1; Institut Camille Jordan, UMR 5208 du CNRS; \\ 43, boulevard du 11 novembre 1918, F-69622 Villeurbanne Cedex, France \\ zeng@math.univ-lyon1.fr, http://math.univ-lyon1.fr/ ${ }^{\sim}$ zeng
}

Submitted: Apr 8, 2012; Accepted: Jun 30, 2012; Published: Jul 12, 2012

Mathematics Subject Classifications: 05A10, 05A30, 11B65

\begin{abstract}
We propose an elemantary approach to Zudilin's q-question about Schmidt's problem [Electron. J. Combin. 11 (2004), \#R22], which has been solved in a previous paper [Acta Arith. 127 (2007), 17-31]. The new approach is based on a $q$-analogue of our recent result in [J. Number Theory 132 (2012), 1731-1740] derived from $q$-Pfaff-Saalschütz identity.
\end{abstract}

\section{Introduction}

In 2007, answering a question of Zudilin [7], the following result was proved in [3].

Theorem 1.1. Let $r \geqslant 1$. Then there exists a unique sequence of polynomials $\left\{c_{i}^{(r)}(q)\right\}_{i=0}^{\infty}$ in $q$ with nonnegative integral coefficients such that, for any $n \geqslant 0$,

$$
\sum_{k=0}^{n} q^{r\left(\begin{array}{c}
n-k \\
2
\end{array}\right)+(1-r)\left(\begin{array}{l}
n \\
2
\end{array}\right)}\left[\begin{array}{c}
n \\
k
\end{array}\right]^{r}\left[\begin{array}{c}
n+k \\
k
\end{array}\right]^{r}=\sum_{i=0}^{n} q^{\left(\begin{array}{c}
n-i \\
2
\end{array}\right)+(1-r)\left(\begin{array}{c}
i \\
2
\end{array}\right)}\left[\begin{array}{c}
n \\
i
\end{array}\right]\left[\begin{array}{c}
n+i \\
i
\end{array}\right] c_{i}^{(r)}(q) .
$$

Here, the $q$-binomial coefficients $\left[\begin{array}{l}n \\ k\end{array}\right]$ are defined by

$$
\left[\begin{array}{l}
n \\
k
\end{array}\right]= \begin{cases}\frac{(q)_{n}}{(q)_{k}(q)_{n-k}}, & \text { if } 0 \leqslant k \leqslant n \\
0, & \text { otherwise }\end{cases}
$$

where $(q)_{0}=1$ and $(q)_{n}=(1-q)\left(1-q^{2}\right) \cdots\left(1-q^{n}\right)$ for $n=1,2, \ldots$ It is well known that $\left[\begin{array}{l}n \\ k\end{array}\right]$ is a polynomial in $q$ with nonnegative integral coefficients of degree $k(n-k)$ (see [2, p. 33]). 
The proof of (1.1) given in [3] is a $q$-analogue of Zudilin's [7] approach to Schmidt's problem (see $[5,6]$ ) by first using the $q$-Legendre inversion formula to obtain a formula for $c_{k}^{(r)}(q)$ and then applying a basic hypergeometric identity due to Andrews [1] to show that the latter expression is indeed a polynomial in $q$ with nonnegative integral coefficients. In this paper, we shall propose a new and elementary approach to Zudilin's q-question, which yields not only a new proof of Theorem 1.1, but also more solutions to Zudilin's $q$-question about Schmidt's problem.

Our starting point is the following $q$-version of Lemma 4.2 in [4].

Lemma 1.2. Let $k \geqslant 0$ and $r \geqslant 1$. Then there exists a unique sequence of Laurent polynomials $\left\{P_{k, i}^{(r)}(q)\right\}_{i=k}^{r k}$ in $q$ with nonnegative integral coefficients such that, for any $n \geqslant k$,

$$
\left[\begin{array}{l}
n \\
k
\end{array}\right]^{r}\left[\begin{array}{c}
n+k \\
k
\end{array}\right]^{r}=\sum_{i=k}^{\min \{n, r k\}} q^{(r k-i) n}\left[\begin{array}{c}
n \\
i
\end{array}\right]\left[\begin{array}{c}
n+i \\
i
\end{array}\right] P_{k, i}^{(r)}(q) .
$$

Moreover, the polynomials $P_{k, i}^{(r)}(q)$ can be computed recursively by $P_{k, k}^{(1)}(q)=1$ and

$$
P_{k, k+j}^{(r+1)}(q)=\sum_{i=k}^{r k} q^{(j-i)(j+k)}\left[\begin{array}{c}
k+i \\
i
\end{array}\right]\left[\begin{array}{c}
k \\
i-j
\end{array}\right]\left[\begin{array}{c}
k+j \\
j
\end{array}\right] P_{k, i}^{(r)}(q), 0 \leqslant j \leqslant r k .
$$

To derive Theorem 1.1 from Lemma 1.2 we first consider a more general problem. Let $f(x, y)$ and $g(x, y)$ be any polynomials in $x$ and $y$ with integral coefficients. Multiplying (1.2) by $q^{-n k r+f(k, r)}$ and summing over $k$ from 0 to $n$ we obtain

$$
\sum_{k=0}^{n} q^{-n k r+f(k, r)}\left[\begin{array}{l}
n \\
k
\end{array}\right]^{r}\left[\begin{array}{c}
n+k \\
k
\end{array}\right]^{r}=\sum_{i=0}^{n} q^{-n i-g(i, r)}\left[\begin{array}{l}
n \\
i
\end{array}\right]\left[\begin{array}{c}
n+i \\
i
\end{array}\right] \sum_{k=0}^{i} T_{k, i}^{(r)}(q),
$$

where

$$
T_{k, i}^{(r)}(q)=q^{f(k, r)+g(i, r)} P_{k, i}^{(r)}(q), 0 \leqslant k \leqslant i \text {, and } P_{k, i}^{(r)}(q)=0 \text { if } i>k r .
$$

Obviously, $T_{k, i}^{(r)}(q)$ are Laurent polynomials in $q$ with nonnegative integral coefficients. For example, taking $f=g=0$, we immediately obtain the following result.

Theorem 1.3. Let $r \geqslant 1$. Then there exists a unique sequence of Laurent polynomials $\left\{b_{i}^{(r)}(q)\right\}_{i=0}^{\infty}$ in $q$ with nonnegative integral coefficients such that, for any $n \geqslant 0$,

$$
\sum_{k=0}^{n} q^{-r k n}\left[\begin{array}{l}
n \\
k
\end{array}\right]^{r}\left[\begin{array}{c}
n+k \\
k
\end{array}\right]^{r}=\sum_{i=0}^{n} q^{-n i}\left[\begin{array}{c}
n \\
i
\end{array}\right]\left[\begin{array}{c}
n+i \\
i
\end{array}\right] b_{i}^{(r)}(q) .
$$

Moreover, we have $b_{i}^{(r)}(q)=\sum_{k=0}^{i} P_{k, i}^{(r)}(q)$. 
Now, we look for a sufficient condition for $T_{k, i}^{(r)}(q)$ in $(1.4)$ to be a polynomial. It follows from (1.3) that

$$
T_{k, i}^{(r+1)}(q)=\sum_{j=k}^{r k} q^{A}\left[\begin{array}{c}
k+j \\
j
\end{array}\right]\left[\begin{array}{c}
k \\
i-j
\end{array}\right]\left[\begin{array}{l}
i \\
k
\end{array}\right] T_{k, j}^{(r)}(q),
$$

where

$$
A=f(k, r+1)+g(i, r+1)-f(k, r)-g(j, r)+i(i-k-j) .
$$

Hence, the positivity of $A$ will ensure that $T_{k, i}^{(r)}(q)$ is a polynomial in $q$.

We shall first prove Lemma 1.2 in the next section and then prove Theorem 1.1 in Section 3 by choosing special polynomials $f$ and $g$. Some open problems are raised in Section 4.

\section{Proof of Lemma 1.2}

We proceed by induction on $r$. We need the following form of Jackson's $q$-Pfaff-Saalschütz identity (see [2, pp. 37-38] or [5] for example):

$$
\left[\begin{array}{c}
m+n \\
M
\end{array}\right]\left[\begin{array}{c}
n \\
N
\end{array}\right]=\sum_{j \geqslant 0} q^{(N-j)(M-m-j)}\left[\begin{array}{c}
M-m \\
j
\end{array}\right]\left[\begin{array}{c}
N+m \\
m+j
\end{array}\right]\left[\begin{array}{c}
m+n+j \\
M+N
\end{array}\right] .
$$

Substituting $m \rightarrow k-i, n \rightarrow n+i, M \rightarrow n-i$ and $N \rightarrow i$ in (2.1), we get

$$
\left[\begin{array}{c}
n+k \\
n-i
\end{array}\right]\left[\begin{array}{c}
n+i \\
i
\end{array}\right]=\sum_{j=0}^{i} q^{(i-j)(n-k-j)}\left[\begin{array}{c}
n-k \\
j
\end{array}\right]\left[\begin{array}{c}
k \\
i-j
\end{array}\right]\left[\begin{array}{c}
n+k+j \\
n
\end{array}\right],
$$

which can be rewritten as

$$
\left[\begin{array}{c}
n \\
i
\end{array}\right]\left[\begin{array}{c}
n+i \\
i
\end{array}\right]=\sum_{i=0}^{i} q^{(i-j)(n-k-j)} \frac{(q)_{k+i}(q)_{j}}{(q)_{k+j}(q)_{i}}\left[\begin{array}{c}
k \\
i-j
\end{array}\right]\left[\begin{array}{c}
n-k \\
j
\end{array}\right]\left[\begin{array}{c}
n+k+j \\
j
\end{array}\right] .
$$

It is clear that (1.2) holds for $r=1$ with $P_{k, k}^{(r)}(q)=1$. Suppose that (1.2) holds for some $r \geqslant 1$. Multiplying both sides of (1.2) by $\left[\begin{array}{l}n \\ k\end{array}\right]\left[\begin{array}{c}n+k \\ k\end{array}\right]$ and applying (2.2), we immediately get

$$
\begin{aligned}
{\left[\begin{array}{l}
n \\
k
\end{array}\right]^{r+1}\left[\begin{array}{c}
n+k \\
k
\end{array}\right]^{r+1}=} & \sum_{i=k}^{r k} q^{(r k-i) n}\left[\begin{array}{l}
n \\
k
\end{array}\right]\left[\begin{array}{c}
n+k \\
k
\end{array}\right] P_{k, i}^{(r)}(q) \\
& \times \sum_{j=0}^{i} q^{(i-j)(n-k-j)} \frac{(q)_{k+i}(q)_{j}}{(q)_{k+j}(q)_{i}}\left[\begin{array}{c}
k \\
i-j
\end{array}\right]\left[\begin{array}{c}
n-k \\
j
\end{array}\right]\left[\begin{array}{c}
n+k+j \\
j
\end{array}\right] \\
= & \sum_{j=0}^{r k} q^{(r k-j) n}\left[\begin{array}{c}
n \\
k+j
\end{array}\right]\left[\begin{array}{c}
n+k+j \\
k+j
\end{array}\right] P_{k, k+j}^{(r+1)}(q),
\end{aligned}
$$

where $P_{k, k+j}^{(r+1)}(q)$ is given by (1.3). By the induction hypothesis, these $P_{k, k+j}^{(r+1)}(q)$ are Laurent polynomials in $q$ with nonnegative integral coefficients. Hence Lemma 1.2 is true for $r+1$. 


\section{Proof of Theorem 1.1}

In $(1.4)$, taking $f(k, r)=r\left(\begin{array}{c}k+1 \\ 2\end{array}\right), g(i, r)=(r-2)\left(\begin{array}{c}i \\ 2\end{array}\right)-i$, and multiplying by $q^{\left(\begin{array}{c}n \\ 2\end{array}\right) \text {, we }}$ obtain (1.1) with

$$
c_{i}^{(r)}(q)=q^{(r-2)\left(\begin{array}{c}
i \\
2
\end{array}\right)-i} \sum_{k=0}^{i} q^{r\left(\begin{array}{c}
k+1 \\
2
\end{array}\right)} P_{k, i}^{(r)}(q) .
$$

By (1.8), the corresponding $A$ reads as follows:

$$
A=(r-2)\left[\left(\begin{array}{l}
i \\
2
\end{array}\right)-\left(\begin{array}{l}
j \\
2
\end{array}\right)\right]+\left(\begin{array}{c}
i-k \\
2
\end{array}\right)+(i-1)(i-j) .
$$

If $r \geqslant 2$, since $i \geqslant j$, we have $A \geqslant 0$. If $r=1$, then (1.7) implies that $j=k$ and $A=2\left(\begin{array}{c}i-k \\ 2\end{array}\right) \geqslant 0$. Thus $c_{i}^{(r)}(q)$ in (3.1) is a polynomial in $q$. For example, by (1.5) we have

$$
T_{k, i}^{(2)}(q)=q^{2\left(\begin{array}{c}
i-k \\
2
\end{array}\right)}\left[\begin{array}{c}
2 k \\
i
\end{array}\right]\left[\begin{array}{c}
i \\
k
\end{array}\right]^{2}
$$

and

$$
c_{i}^{(2)}(q)=\sum_{k=0}^{i} q^{2\left(\begin{array}{c}
i-k \\
2
\end{array}\right)}\left[\begin{array}{c}
2 k \\
i
\end{array}\right]\left[\begin{array}{c}
i \\
k
\end{array}\right]^{2}
$$

which coincides with $[3,(3,1)]$.

\section{Open problems}

For any positive integers $r$ and $s$, it is easy to see that there are uniquely determined rational numbers $c_{k}^{(r, s)}(k \geqslant 0)$, independent of $n(n \geqslant 0)$, satisfying

$$
\sum_{k=0}^{n}\left(\begin{array}{l}
n \\
k
\end{array}\right)^{r}\left(\begin{array}{c}
n+k \\
k
\end{array}\right)^{r}=\sum_{k=0}^{n}\left(\begin{array}{l}
n \\
k
\end{array}\right)^{s}\left(\begin{array}{c}
n+k \\
k
\end{array}\right)^{s} c_{k}^{(r, s)}
$$

When $s=1$ and $r \geqslant 1$, the integrality of $c_{k}^{(r, s)}$ is the original problem of Schmidt [5]. When $s>1$ and $r>s$, we observe that the numbers $c_{k}^{(r, s)}$ are not always integers. From arithmetical point of view, the following problems may be interesting.

Conjecture 4.1. For any $s>1$ and $n \geqslant 0$, there is an integer $r>s$ such that all the numbers $c_{k}^{(r, s)}(0 \leqslant k \leqslant n)$ are integers.

For $s=2$, via Maple, we find that the least such integers $r:=r(n, s)$ are $r(0,2)=$ $r(1,2)=r(2,2)=3, r(3,2)=7, r(4,2)=32, r(5,2)=212$.

Conjecture 4.2. For any $r>s>1$, there is a positive integer $n$ such that $c_{n}^{(r, s)}$ is not an integer.

Acknowledgments. This work was partially supported by the Fundamental Research Funds for the Central Universities. 


\section{References}

[1] G.E. Andrews, Problems and prospects for basic hypergeometric functions, In: The Theory and Application of Special Functions, R. Askey, Ed., Academic Press, New York, 1975, pp. 191-224.

[2] G.E. Andrews, The Theory of Partitions, Cambridge University Press, Cambridge, 1998.

[3] V.J.W. Guo, F. Jouhet, and J. Zeng, Factors of alternating sums of products of binomial and q-binomial coefficients, Acta Arith. 127 (2007), 17-31.

[4] V.J.W. Guo, J. Zeng, Proof of some conjectures of Z.-W. Sun on congruences for Apéry polynomials, J. Number Theory 132 (2012), 1731-1740.

[5] A.L. Schmidt, Generalized q-Legendre polynomials, J. Comput. Appl. Math. 49 (1993), 243-249.

[6] V. Strehl, Binomial identities — combinatorial and algorithmical aspects, Discrete Math. 136 (1994), 309-346.

[7] W. Zudilin, On a combinatorial problem of Asmus Schmidt, Electron. J. Combin. 11 (2004), \#R22. 